PROCEEDINGS OF THE

AMERICAN MATHEMATICAL SOCIETY

Volume 134, Number 10, October 2006, Pages 2989-2996

S 0002-9939(06)08392-4

Article electronically published on May 5, 2006

\title{
ASYMPTOTIC PHASE AND INVARIANT FOLIATIONS NEAR PERIODIC ORBITS
}

\author{
FREDDY DUMORTIER \\ (Communicated by Carmen C. Chicone)
}

\begin{abstract}
The paper deals with asymptotic phase and invariant foliations near periodic orbits, extending for two-dimensional smooth vector fields results that have been obtained by Chicone and Liu (2004). The problem of the existence of asymptotic phase is completely solved for analytic vector fields and is reduced to a problem of infinite codimension for $C^{\infty}$ systems. Moreover it is proven that whenever asymptotic phase occurs, or in other words, when the periodic orbit is isochronous, then there also exists a $C^{\infty}$ foliation, with leaves transversally cutting the periodic orbit and invariant under the flow of the vector field. The paper also provides some results in three dimensions.
\end{abstract}

\section{INTRODUCTION AND STATEMENT OF THE MAIN RESULTS}

In CL C. Chicone and W. Liu study asymptotic phase for periodic orbits of smooth planar vector fields. Before describing their results and presenting ours, we will recall the definition of asymptotic phase as given in CL.

Let $\Gamma$ be a periodic orbit of a flow $\phi_{t}$, associated to a vector field $X$ defined on some open subset $U$ of $\mathbb{R}^{n}$, or on an open subset $U$ of some $n$-dimensional manifold. A point $q \in U$ has asymptotic phase with respect to $\Gamma$ if there is a point $p \in \Gamma$ such that $\lim _{t \rightarrow \infty} d\left(\phi_{t}(q), \phi_{t}(p)\right)=0$ or $\lim _{t \rightarrow-\infty} d\left(\phi_{t}(q), \phi_{t}(p)\right)=0$, where $d(.,$.$) stands$ for Euclidean distance on $\mathbb{R}^{n}$ or a Riemannian metric on the manifold. In this case, $q$ is said to be in phase with $p$. We also recall that Chicone and Liu call a limit cycle isochronous if there is a neighbourhood of $\Gamma$ such that every point in the neighbourhood is in phase with a point on $\Gamma$. This definition is clearly in agreement with the traditional notion of isochronicity of centers.

As in CL we use the notation $\Sigma$ for any section transversally cutting $\Gamma$ at some $p \in \Gamma, \tau$ denotes the time of first return to $\Sigma$ and $P$ denotes the corresponding Poincaré map, or first return map.

In this paper we will always assume that $X$ and $\Sigma$ are $C^{\infty}$ (respectively $C^{\omega}$ ) so that also $\tau$ and $P$ are $C^{\infty}$ (respectively $C^{\omega}$ ); we let $r$ represent a regular $C^{\infty}$ (respectively $C^{\omega}$ ) parameter on $\Sigma$ with the property that $\{r=0\}$ agrees with $p$,

Received by the editors May 4, 2005.

2000 Mathematics Subject Classification. Primary 34D05, 34C07, 34C20.

Key words and phrases. Periodic orbit, asymptotic phase, isochronous, invariant foliations, Poincaré map, return-time map.

The author thanks the Ministerio de Educacion y Ciencia from Spain for their financial support and the Centre de Recerca Matemàtica at Bellaterra for their hospitality during the preparation of this paper. 
and we express both $P$ and $\tau$ in $r$. Let us first take $n=2$. In case $\Gamma$ is a hyperbolic periodic orbit, the occurrence of asymptotic phase in some neighbourhood of $\Gamma$, linked to the existence of an invariant foliation, is well known (see, e.g., [HPS]).

In $\left[\mathrm{CL}\right.$, Chicone and Liu consider the case when $P^{\prime}(0)=1$ and $P^{\prime \prime}(0) \neq 0$ and show that $\tau^{\prime}(0)=0$ is a necessary and sufficient condition for every point in some neighbourhood of $\Gamma$ to be in phase with a point on $\Gamma$. In fact their result holds for $C^{2}$ vector fields $X$, and they even show that their result is sharp, in a sense that the condition $\tau^{\prime}(0)=0$ is no longer necessary for vector fields $X$ that are merely $C^{1+\alpha}$, and this for whatsoever $\alpha \in(0,1)$.

In the next theorem we present a generalization of this result, partly announced in $\mathrm{CL}$, which completely solves the problem of the occurrence of asymptotic phase for analytic vector fields and reduces it to a problem of infinite codimension for $C^{\infty}$ systems. The proof reveals to be quite short, but it uses a technical theorem, based on heavy calculations, that is however already present in the literature ([DRR $]$ ).

Without stating it precisely, we remark that similar results as in the theorem can be obtained for vector fields $X$ of finite differentiability; the differentiability has to be sufficiently high depending on the precise set of conditions (see, e.g., [DR]).

In the theorem, as well as in the proof in section 2 we will use the $C^{\infty}$ (respectively $C^{\omega}$ ) functions $I(r)=r$ and $T(r)=T$, with $T$ denoting the (prime) period of $\Gamma$. For any $C^{\infty}$ function $f, j_{\infty}(f)(0)$ denotes the $\infty$-jet or Taylor development of $f$ at 0 .

Theorem 1. Let $X$ be a $C^{\infty}$ vector field defined on some open subset $U \subset \mathbb{R}^{2}$ or $U \subset M$, with $M$ some $C^{\infty}$ regular surface. Suppose that $X$ has a periodic orbit $\Gamma \subset U$ having a cylindrical tubular neighbourhood. Let $\Sigma, r, \tau, P, I$ and $T$ be as above.

(1) Suppose that $j_{\infty}(P-I)(0) \neq 0$, and denote by $m$ the smallest natural number with $\frac{d^{m}(P-I)}{d r^{m}}(0) \neq 0$, and by $n$ the smallest natural number such that $\frac{d^{n}(\tau-T)}{d r^{n}}(0) \neq 0$, with the convention that $n=\infty$ if $j_{\infty}(\tau-T)(0)=0$. Then a necessary and sufficient condition for $\Gamma$ to be isochronous is that $n \geq m$. Moreover, in case $\Gamma$ is isochronous there is a $C^{\infty}$ foliation, defined in a neighborhood of $\Gamma$, that cuts $\Gamma$ transversally and is invariant under the flow $\phi_{t}$ of $X$.

(2) If $j_{\infty}(P-I)(0)=0$, then a necessary condition for the existence of some neighbourhood of $\Gamma$ in which every point is in phase with a point on $\Gamma$, is that $j_{\infty}(\tau-T)(0)=0$.

If $\Gamma$ has a Möbius strip as tubular neighbourhood, then the conclusions expressed in (1) and (2) still hold, if we change "first return" in the definition of $P$ and $\tau$ by "second return" and if we take $T$ to express twice the (prime) period of $\Gamma$.

Remarks. For $\Gamma$ with a cyclindrical tubular neighbourhood (as in the case when $\left.U \subset \mathbb{R}^{2}\right)$, and both $j_{\infty}(P-I)(0)=0$ and $j_{\infty}(\tau-T)(0)=0$, it is quite easy to find examples for which there exists a neighbourhood in which every point is in phase with a point on $\Gamma$ as well as examples for which it is not the case. Even for $P=I$ and $j_{\infty}(\tau-T)(0)=0, C^{\infty}$ examples of both kind exist. Of course if $P=I$ and $\tau \equiv T$, then there exists a neighbourhood of $\Gamma$ in which every point is in phase with a point on $\Gamma$ and in which locally a $C^{\infty}$ invariant foliation exists. So the theorem provides a full characterisation for the occurrence of asymptotic phase in analytic 
two-dimensional vector fields $X$. We will give the proof of Theorem 1 in section 2, while in section 3 we will present a few interesting examples in dimension three.

\section{Asymptotic phase in dimension two: Proof of Theorem 1}

We refer to the notations and conditions introduced in section 1 . We will begin by supposing that $\Gamma$ has a cylindrical tubular neighbourhood, as is the case when $U \subset \mathbb{R}^{2}$. The necessary adaptations for a periodic orbit $\Gamma$ with a Möbius strip as tubular neighbourhood will be elaborated at the end of this section.

By the flow box theorem we know that there exist $C^{\infty}$ (respectively $C^{\omega}$ ) coordinates $(x, y)$ in the neighbourhood of $p$ in which $\Gamma$ is situated on $\{y=0\}, \Sigma$ is situated on $\{x=0\}, r$ on $\Sigma$ is equal to $y, X$ is given by $\frac{\partial}{\partial x}$ and $\phi_{T}$ can be expressed as

$$
\Phi:(x, y) \mapsto(\bar{x}, \bar{y})=(x+\tau(y)-T, P(y)) .
$$

In any case $\frac{d P}{d y}(0)>0$. When $\frac{d P}{d y}(0) \neq 1$ the limit cycle $\Gamma$ is hyperbolic and the results, stated in Theorem 1, are well known (see, e.g., HPS and also CL]).

From now on we can suppose that $\frac{d P}{d y}(0)=1$. If $\frac{d \tau}{d y}(0) \neq 0$, it is easy to show that asymptotic phase cannot occur. We will not give the specific argument here, but incorporate it in the more general argument that we will present in a moment. Let us now suppose that $\frac{d P}{d y}(0)=1$ and $\frac{d \tau}{d y}(0)=0$.

We introduce the $C^{\infty}$ (respectively $C^{\omega}$ ) functions $f$ and $g$, defined by

$$
\left\{\begin{array}{l}
\tau(y)=T+y f(y), \\
P(y)=y+y g(y),
\end{array}\right.
$$

so that

$$
\Phi(x, y)=(x+y f(y), y+y g(y))
$$

with $f(0)=g(0)=0$.

We recall a theorem of Takens that we state as Proposition 2.

Proposition $2\left([\mathrm{~T})\right.$. Let $\widetilde{\Psi}$ be the germ at 0 of a $C^{\infty}$ diffeomorphism $\Psi:\left(\mathbb{R}^{2}, 0\right) \rightarrow$ $\left(\mathbb{R}^{2}, 0\right)$ and let $R$ be the semi-simple part of $D \Psi(0)=j_{1}(\Psi)(0)$ with eigenvalues $e^{ \pm 2 \pi i \alpha}$ or \pm 1 . Then there is a unique $\tilde{Y}$, germ at 0 of a $C^{\infty}$ vector field $Y$ on $\mathbb{R}^{2}$ with $Y(0)=0$, invariant under $R$, such that, up to a $C^{\infty}$ change of coordinates, $\widetilde{\Psi}$ is equal to $R \circ \widetilde{Y}_{1}$, where $\widetilde{Y}_{t}$ denotes the formal flow of $\tilde{Y}$. Moreover, for all $k \in \mathbb{N} \cup\{\infty\}$, the $k$-jet of $\widetilde{Y}$ only depends on the $k$-jet of $\widetilde{\Psi}$.

We call $R \circ \widetilde{Y}_{1}$ the formal normal form of $\widetilde{\Psi}$. We apply this theorem to $\widetilde{\Phi}$, germ of $\Phi$ at 0 , knowing that $R=I$, the identity map of $\mathbb{R}^{2}$. We can attach to $\widetilde{\Phi}$ a formal vector field $\widetilde{Y}$, in the sense that $\widetilde{\Phi}=\widetilde{Y}_{1}$. Because of the specific expression (3) of $\Phi, \widetilde{Y}$ will only depend on $y$. By Borel's representation theorem we can realize $\widetilde{Y}$ as the $\infty$-jet at 0 of a $C^{\infty}$ vector field $Y$ with

$$
Y=y\left(F(y) \frac{\partial}{\partial x}+G(y) \frac{\partial}{\partial y}\right) .
$$

We can now rely on a theorem of Belitskii, that can also be found as theorem 2 in DRR including a complete proof. We state it as Proposition 3. 
Proposition 3 ([B], $\overline{\mathrm{DRR}}])$. Let $S \subset M$ be a submanifold of a manifold $M$ and $\Psi$ a $C^{\infty}$ diffeomorphism on $M$ such that $\Psi(z)=z$ for all $z \in S$. Suppose that $\Psi$ is quasi-hyperbolically contracting to $S$ along some compact subset $K \subset S$. If $\Psi^{\prime}$ is a germ of a $C^{\infty}$ diffeomorphism of $M$ along $S$ such that $j_{\infty}\left(\Psi^{\prime}\right)(z)=j_{\infty}(\Psi)(z)$, for all $z \in S$, then we can find a germ of $C^{\infty}$ diffeomorphism $H$ conjugating $\Psi$ and $\Psi^{\prime}$ along $K$ and with the extra property that $j_{\infty}(H)(z)=j_{\infty}(I d)(z)$ for all $z \in S$.

Proposition 3 implies that, along $\{y=0\}$, $\Phi$, as given in (3), is $C^{\infty}$ conjugate to the time 1-mapping of $Y$, as given in (4) when $n \geq m$. However to check the applicability of Proposition 3 we will first recall the definition of "quasi-hyperbolic contraction" and show that $\Phi$ or $\Phi^{-1}$ satisfies that property towards $\{y=0\}$.

Definition. Let $M$ be a $C^{\infty}$ manifold, $S \subset M$ a $C^{\infty}$ submanifold and $\Psi: M \rightarrow M$ a $C^{\infty}$ diffeomorphism with the property that $\Psi(z)=z$ for all $z \in S$. We say that $\Psi$ is "quasi-hyperbolically contracting" to $S$ along a compact subset $K \subset S$, if for some Riemannian metric on $M$, there exists a neighbourhood $V$ of $K$ in $M$, a natural number $k \geq 1$ and a positive constant $a>0$ so that

$$
\begin{gathered}
\|\Psi(z)\|_{S} \leq\|z\|_{S}\left(1-a\|z\|_{S}^{k}\right) \quad \text { for all } \quad z \in V, \\
j_{k}(\Psi)(z)=j_{k}(I d)(z) \quad \text { for all } \quad z \in S,
\end{gathered}
$$

with $\|m\|_{S}$ denoting the distance from $m \in V$ to $S$ in the chosen Riemannian metric.

The existence of the inequality in the definition does not depend on the choice of Riemannian metric, and both conditions only depend on the germ of $\Psi$ along $K$.

In view of the expression for $\Phi$, as given in (3), it is easy to see that $\Phi$, or $\Phi^{-1}$, is a quasi-hyperbolic contraction to $\{y=0\}$ along any compact subset $K \subset\{y=0\}$, if we suppose that $j_{\infty}(P-I)(0) \neq 0$ and that $n \geq m$, with $n$ and $m$ as defined in statement (1) of Theorem 1. As such, in appropriate $C^{\infty}$ coordinates near $(x, y)=(0,0)$, obtained after a $C^{\infty}$ coordinate change that is $\infty$-close to the identity along $\{y=0\}$, we can suppose that $\Phi$ is given by the time 1-map of a $C^{\infty}$ vector field $Y$ with an expression as in (4). Knowing that $n \geq m$ and that $m$ is the smallest natural number with $\frac{d^{m}(P-I)}{d y^{m}}(0) \neq 0$, we can write $Y$ as

$$
Y=y^{m}\left(\bar{F}(y) \frac{\partial}{\partial x}+\bar{G}(y) \frac{\partial}{\partial y}\right)
$$

for some $C^{\infty}$ functions $\bar{F}$ and $\bar{G}$ with $\bar{G}(0) \neq 0$. The orbits of $\bar{Y}$, with

$$
\bar{Y}=\bar{F}(y) \frac{\partial}{\partial x}+\bar{G}(y) \frac{\partial}{\partial y},
$$

define a $C^{\infty}$ foliation, whose leaves transversally cut $\{y=0\}$. The natural saturation of this foliation by means of the flow maps $\phi_{t}$ defines an invariant $C^{\infty}$ foliation and at the same time induces the existence of neighbourhoods of $\Gamma$, all of whose points are in asymptotic phase with points of $\Gamma$.

Continuing with the orientable case, i.e. the case in which $\Gamma$ has cylindrical neighbourhoods, we will now show that if we violate the condition " $j_{\infty}(P-I)(0) \neq$ $0, n \geq m$ ", then $\Gamma$ cannot be isochronous. We therefore again write $\Phi$ as in (3), with $g(0)=0$, but accepting also that $f(0) \neq 0$, to include in our treatment the case in which $\frac{d P}{d y}(0)=1$ and $\frac{d \tau}{d y}(0) \neq 0$. 
Considering the smallest natural number $n$ for which $\frac{d^{n}(\tau-T)}{d y^{n}}(0) \neq 0$, we can write $\Phi$ as

$$
\begin{aligned}
& \bar{x}=x+y^{n}(c+k(y)), \\
& \bar{y}=y+y^{n+1} \ell(y)
\end{aligned}
$$

for some $c \neq 0$, and some $C^{\infty}$ functions $k$ and $\ell$ with $k(0)=0$.

We can suppose that the $X$-orbits near $\Gamma$ stay at a bounded distance from $\Gamma$ for $t \rightarrow \infty$; if not we will use $\Phi^{-1}$ instead of $\Phi$. Let us now first suppose that $c>0$ and consider some $d>0$, sufficiently small (to be specified in a moment); we also restrict to $y>0$. We will check that for $d$ sufficiently small the function $y e^{x / d}$ serves as a "Lyapunov function" for $\Phi$, in a way that points $z$ on $\left\{y=\alpha e^{-x / d}\right\}$, with $\alpha>0$ sufficiently small, having their image above that curve, obstructing the possibility for the iterates $\Phi^{n}(z)=\phi_{n T}(z)$ to converge to some point of $\Gamma$.

To check this we calculate, for $\left(\bar{x}_{0}, \bar{y}_{0}\right)=\Phi\left(x_{0}, y_{0}\right)$, with $y_{0}>0$, the quantity

$$
\begin{aligned}
& \left|\begin{array}{cc}
1 & \bar{x}_{0}-x_{0} \\
-\frac{\alpha}{d} e^{-x_{0} / d} & \bar{y}_{0}-y_{0}
\end{array}\right|=\left|\begin{array}{cc}
1 & y_{0}^{n}\left(c+k\left(y_{0}\right)\right) \\
-\frac{\alpha}{d} e^{-x_{0} / d} & y_{0}^{n+1} \ell\left(y_{0}\right)
\end{array}\right| \\
= & y_{0}^{n}\left[\frac{\alpha}{d} e^{-x_{0} / d}\left(c+k\left(y_{0}\right)\right)+y_{0} \ell\left(y_{0}\right)\right] \\
= & y_{0}^{n+1}\left[\frac{1}{d}\left(c+k\left(y_{0}\right)\right)+\ell\left(y_{0}\right)\right]
\end{aligned}
$$

which is clearly strictly positive for $y_{0}>0$ sufficiently small, if we take $d$ sufficiently small.

In case $c<0$ we can, for $y>0$, make a similar reasoning based on the "Lyapunov function" $y e^{-x / d}$ with $d>0$ sufficiently small. Analogous adaptations can be made on the side $\{y<0\}$.

It remains to prove the statements concerning a periodic orbit $\Gamma$ having Möbiusstrips as neighbourhoods. The argumentation given in the orientable case, to show that $\Gamma$ cannot be isochronous under the cited conditions, can obviously be used, without change, in the non-orientable case if we use "second return" in the definition of $P$ and $\tau$ instead of "first return".

For the affirmative statements concerning the isochronicity of $\Gamma$ we consider both the first return map, that we denote by $P_{1}$, and the second return map, that we denote by $P_{2}$, as well as the time of first return, denoted by $\tau_{1}$, and the time of second return, denoted by $\tau_{2}$. We have

$$
P_{2}(r)=P_{1}\left(P_{1}(r)\right) \text { and } \tau_{2}(r)=\tau_{1}(r)+\tau_{1}\left(P_{1}(r)\right) .
$$

From the first equation of (8) we see that $P_{1}^{\prime}(0)=-1$ if and only if $P_{2}^{\prime}(0)=1$. We can restrict to treating $P_{1}^{\prime}(0)=-1$, since otherwise $\Gamma$ is hyperbolic and the required results are well known [HPS].

If we write $P_{1}(r)=-r\left(1+a r^{k}+o\left(r^{k}\right)\right)$, we see that

$$
P_{2}(r)=\left(1+a\left(r^{k}+(-r)^{k}\right)+o\left(r^{k}\right)\right) \text {. }
$$


It makes it clear that $m$ in Theorem 1 has to be odd, while $m$ is also the smallest natural number such that

$$
\frac{d^{m}\left(P_{1}+I\right)}{d r^{m}}(0) \neq 0
$$

Using the second equation of (8), still requiring that $P_{1}^{\prime}(0)=-1$, and supposing that $\tau_{1}(r)=T+b r^{n}+o\left(r^{n}\right)$, we see that

$$
\tau_{2}(r)=2 T+b\left(r^{n}+(-r)^{n}\right)+o\left(r^{n}\right)
$$

implying that $n$ in Theorem 1 has to be even, and that it is also the smallest natural number such that

$$
\frac{d^{n}\left(\tau_{1}-T\right)}{d r^{n}}(0) \neq 0
$$

where this time $T$ represents the (prime) period of $\Gamma$.

Exactly as in the orientable case of the theorem, we can now express $\phi_{T}$ in flow box coordinates near $p \in \Gamma$ and denote it by $\Phi$. The semi-simple part of $D \Phi(0)$ is the linear mapping $R$ with

$$
R(x, y)=(x,-y)
$$

and the formal normal form of $\Phi$ at 0 is given by $R \circ \widetilde{Y}_{1}$ with $\tilde{Y}$ a formal vector field in $y$ which is invariant under $R$. By Borel's representation theorem we can realize $\tilde{Y}$ as the $\infty$-jet at 0 of a $C^{\infty}$ vector field $Y$ as in (4), which moreover satisfies $R_{*} Y=Y$

We know that for some odd $m$, we can write $Y$ as in (5), with the property that the related $\bar{Y}$, as defined in (6), satisfies

$$
R_{*} \bar{Y}=-\bar{Y} .
$$

Expression (9) means that $\bar{Y}$ is time-reversible under $R$, with $R(x, y)=(x,-y)$. Because $\bar{G}(0) \neq 0$, the $\bar{Y}$-orbits will all cut the $y$-axis transversally. As a conclusion we see that the $C^{\infty}$ foliation, given by the $\bar{Y}$-orbits, is invariant under $R$ and hence also under $\phi_{T}$. The natural saturation of this foliation by means of the flow maps $\phi_{t}$ hence defines an invariant $C^{\infty}$ foliation, and as such induces the existence of neighbourhoods of $\Gamma$, all of whose points are in asymptotic phase with points of $\Gamma$; in other words, $\Gamma$ is isochronous.

\section{Asymptotic phase in Dimension three: Examples}

Although no longer restricting to two dimensions, we will nevertheless in this section restrict to periodic orbits $\Gamma$ that are attractors, in the sense that there exists a neighbourhood $V$ of $\Gamma$ in which every orbit tends to $\Gamma$ for $t \rightarrow+\infty$. It is of course very easy to provide examples where all points in the basin of attraction of $\Gamma$ are in asymptotic phase with some point of $\Gamma$, as well as examples where no point, outside $\Gamma$, is in asymptotic phase with some point of $\Gamma$. The following two examples in $\mathbb{R}^{3}$ provide hybrid situations clearly showing that a simple general statement concerning the existence of asymptotic phase in dimensions greater than two is not to be expected. In the first example we obtain that all points outside some given plane $P$ are in asymptotic phase with some point of $\Gamma$, while this does not hold for the points in $P \backslash \Gamma$. In the second example the points that are in asymptotic phase with some point of $\Gamma$ exactly form a plane $P$.

To be in asymptotic phase with some point of $\Gamma$ is hence neither an open property, nor a closed one. The examples are given on $S^{1} \times \mathbb{R}^{2}$, with $S^{1} \times\{0\}$ as closed orbit $\Gamma$; we use $(\theta, r, s)$ as coordinates near it. 


\section{Example 1.}

$$
\left\{\begin{array}{l}
\dot{\theta}=1+r, \\
\dot{s}=-s\left(s^{2}+r^{2}\right), \\
\dot{r}=-4 r\left(s^{2}+r^{2}\right) .
\end{array}\right.
$$

The orbits are situated in the surfaces $\{s=0\}$ and $\left\{r=b s^{4}\right\}$ for some $b \in \mathbb{R}$. In $\{s=0\}$ the equation is given by

$$
\left\{\begin{array}{l}
\dot{\theta}=1+r \\
\dot{r}=-4 r^{3}
\end{array}\right.
$$

and because of Theorem 1 no orbit outside $\Gamma$ can be in asymptotic phase with a point of $\Gamma$. If we restrict to $\left\{r=b s^{4}\right\}$, for some $b \in \mathbb{R}$, then the orbits can be described in $(\theta, s)$-coordinates by

$$
\left\{\begin{array}{l}
\dot{\theta}=1+b s^{4} \\
\dot{s}=-s^{3}\left(1+b^{2} s^{6}\right)
\end{array}\right.
$$

and again by Theorem 1 they are clearly in asymptotic phase with some point of $\Gamma$.

\section{Example 2.}

$$
\left\{\begin{array}{l}
\dot{\theta}=1+r, \\
\dot{r}=-r\left(r^{2}+s^{2}\right), \\
\dot{s}=-s\left(r^{2}+s^{2}\right) .
\end{array}\right.
$$

The orbits are situated in the surfaces $\{r=0\}$ and $\{s=a r\}$ for some $a \in \mathbb{R}$. In $\{r=0\}$ the equation is given by

$$
\left\{\begin{array}{l}
\dot{\theta}=1, \\
\dot{s}=-s^{3},
\end{array}\right.
$$

and clearly all orbits are in asymptotic phase with some piont of $\Gamma$. If we restrict to $\{s=a r\}$, for some $a \in \mathbb{R}$, then the orbits can be described in $(\theta, r)$-coordinates by

$$
\left\{\begin{array}{l}
\dot{\theta}=1+r, \\
\dot{r}=-r^{3}\left(1+a^{2}\right),
\end{array}\right.
$$

and because of Theorem 1 no orbit outside $\Gamma$ can be in asymptotic phase with some point of $\Gamma$.

\section{REFERENCES}

[B] G.R. Belitskii, Functional equations and local conjugacy of mappings of class $C^{\infty}$, Math. USSR Sb. 20, 4 (1973), 587-602.

[CL] C. Chicone, W. Liu, Asymptotic phase revisited, J. Differential Equations, 204 (2004), 227-246. MR2076165 (2005e:34085)

[DR] F. Dumortier, R. Roussarie, Germes de difféomorphismes et de champs de vecteurs en classe de différentiabilité finie (Germs of diffeomorphisms and of vector fields in a finite differentiability class), Ann. Inst. Fourier, Grenoble 33 no.1, (1983), 195-267. MR0698855 (84m:58114)

[DRR] F. Dumortier, P. Rodrigues, R. Roussarie, Germs of diffeomorphisms in the plane, Lecture Notes in Mathematics, vol. 902 Springer-Verlag, Berlin-New York, 1981. MR0653474 (83f:58008) 
[HPS] M. Hirsch, C.C. Pugh, M. Shub, Invariant manifolds, Lecture Notes in Mathematics, vol. 583 Springer-Verlag, Berlin-Heidelberg-New York, 1977. MR0501173 (58:18595)

[T] F. Takens, Forced oscillations and bifurcations, Comm. of the Math. Inst. Rijksuniversiteit Utrecht 3 (1974), 1-59; and Global analysis of dynamical systems, Inst. Phys., Bristol, (2001), 1-61. MR0478235 (57:17720) MR1858470(2002i:37081)

Universiteit Hasselt, Campus Diepenbeek, Agoralaan - Gebouw D, B-3590 Diepenbeek, BELGiUm

E-mail address: freddy.dumortier@uhasselt.be 